\title{
Supplementary Materials: Martingale Difference Divergence Matrix and Its Application to Dimension Reduction for Stationary Multivariate Time Series
}

\author{
Chung Eun Lee And Xiaofeng Shao \\ Department of Statistics \\ University of Illinois at Urbana-Champaign \\ Champaign, Illinois 61820
}

The following supplementary materials contain proofs of Lemma 3.1, Theorem 3.1, Theorem 4.1, and Propositions 4.1.

Proof of Lemma 3.1: For $j, k=1, \cdots, p$,

$$
\begin{aligned}
G_{j}(s) G_{k}(s)^{*} & =E\left[\left(V_{j}-E\left(V_{j}\right)\right) e^{i<s, U>}\right] E\left[\left(V_{k}^{\prime}-E\left(V_{k}^{\prime}\right)\right) e^{-i<s, U^{\prime}>}\right] \\
& =E\left[\left(V_{j}-E\left(V_{j}\right)\right)\left(V_{k}^{\prime}-E\left(V_{k}^{\prime}\right)\right) e^{i<s, U-U^{\prime}>}\right] \\
& =-E\left[\left(V_{j}-E\left(V_{j}\right)\right)\left(V_{k}^{\prime}-E\left(V_{k}^{\prime}\right)\right)\left(1-\cos \left(s<U-U^{\prime}>\right)\right)\right]+A
\end{aligned}
$$

with A representing the term that vanishes when the integral is evaluated. Integrating the above term and using Lemma 1 in Székeley et al. (2007), we can derive that

$$
M D D M_{j k}(V \mid U)=-E\left[\left(V_{j}-E\left(V_{j}\right)\right)\left(V_{k}^{\prime}-E\left(V_{k}^{\prime}\right)\right)\left|U-U^{\prime}\right|_{q}\right]
$$

Therefore, $M D D M(V \mid U)=-E\left[(V-E(V))\left(V^{\prime}-E\left(V^{\prime}\right)\right)^{T}\left|U-U^{\prime}\right|_{q}\right]$.

Proof of Theorem 3.1: The first assertion is a direct consequence of Lemma 3.1. Regarding the second one, let $m=\left(m_{1}, \cdots, m_{p}\right)^{T} \in R^{p}, m \neq 0$, and $Z=m^{T} V$ be a linear combination of $V$ that satisfies $E(Z \mid U)=E(Z)$, then $\operatorname{MDDM}(Z \mid U)=0$ and $m^{T} M D D M(V \mid U) m=M D D M(Z \mid U)=0$, implying that $M D D M(V \mid U)$ is singular. On the other hand, assume that $\operatorname{MDDM}(V \mid U)$ is singular and $m$ is in its null space, i.e., $M D D M(V \mid U) m=0$. Since $M D D M(V \mid U)$ is positive semidefinite, we have $m^{T} M D D M(V \mid U) m=M D D M\left(m^{T} V \mid U\right)=0$, which implies that $E\left(m^{T} V \mid U\right)=$ $E\left(m^{T} V\right)$, i.e., a linear combination of $V$ is conditionally mean independent of $U$. The conclusion follows. 
Proof of Theorem 4.1: We shall treat the case $k_{0}=1$ only as the more general case can be handled in a similar fashion but at the expense of lengthy details. The main idea of the proof is to use Lemma A.1 in Kneip and Utikal (2001), which quantifies the changes of eigenvalues and eigenvectors when passing from a matrix $C$ to a perturbed matrix $C+E$. In our setting, we let $C=\Gamma_{1}=M D D M\left(Y_{t} \mid Y_{t-1}\right)$, and $E=\widehat{\Gamma}_{1}-\Gamma_{1}$. Then for $j=1, \cdots, s$, we get by applying part (a) of that lemma that

$$
\widehat{\lambda}_{j}-\lambda_{j}=\operatorname{tr}\left(\gamma_{j} \gamma_{j}^{T}\left\{\widehat{\Gamma}_{1}-\Gamma_{1}\right\}\right)+R_{1},
$$

where $\left|R_{1}\right| \leq \frac{6 \mid \widehat{\Gamma}_{1}-\Gamma_{1} \|_{2}^{2}}{\min _{\lambda \in E G\left(\Gamma_{1}\right), \lambda \neq \lambda_{j}}\left|\lambda-\lambda_{j}\right|}$. Here $E G(C)=\left(\lambda_{1}(C), \cdots, \lambda_{p}(C)\right)$ denotes the set of eigenvalues of the $p \times p$ matrix $C$. To obtain the order of $\widehat{\lambda}_{j}-\lambda_{j}$, we shall show that

$$
\left\|\widehat{\Gamma}_{1}-\Gamma_{1}\right\|_{2}^{2}=O_{p}\left(n^{-1}\right)
$$

Note that

$$
\begin{aligned}
\left\|\widehat{\Gamma}_{1}-\Gamma_{1}\right\|_{2}^{2} & \leq\left\|\widehat{\Gamma}_{1}-\Gamma_{1}\right\|_{F}^{2} \\
& \leq \sum_{i=1}^{p} \sum_{j=1}^{p}\left|M D D M_{n}\left(Y_{t} \mid Y_{t-1}\right)_{i j}-\operatorname{MDDM}\left(Y_{t} \mid Y_{t-1}\right)_{i j}\right|^{2}
\end{aligned}
$$

Let $Y_{t}=\left(Y_{1, t}, Y_{2, t}, \cdots, Y_{p, t}\right)^{T}$. Write

$$
\begin{aligned}
M D D M_{n}\left(Y_{t} \mid Y_{t-1}\right) & =-\frac{1}{(n-1)^{2}} \sum_{t_{1}=2}^{n} \sum_{t_{2}=2}^{n}\left(Y_{t_{1}}-\bar{Y}_{n-1}\right)\left(Y_{t_{2}}-\bar{Y}_{n-1}\right)^{T}\left|Y_{t_{1}-1}-Y_{t_{2}-1}\right|_{p} \\
M D D M_{n}\left(Y_{t} \mid Y_{t-1}\right)_{i, j} & =\frac{(n-2)}{(n-1)}\left\{\left(U_{1, n}\right)_{i, j}+\left(U_{2, n}\right)_{i, j}+\left(U_{3, n}\right)_{i, j}+\left(U_{4, n}\right)_{i, j}\right\},
\end{aligned}
$$

where $\bar{Y}_{n-1}=(n-1)^{-1} \sum_{t=2}^{n} Y_{t}$, and $M D D M_{n}\left(Y_{t} \mid Y_{t-1}\right)_{i, j}$ is the $(i, j)$ th entry of $M D D M_{n}\left(Y_{t} \mid Y_{t-1}\right)$. Furthermore,

$$
\begin{gathered}
\left(U_{1, n}\right)_{i j}=-\frac{1}{(n-1)(n-2)} \sum_{t_{1}=2}^{n} \sum_{t_{2} \neq t_{1}}\left(Y_{i, t_{1}}-E\left(Y_{i, t_{1}}\right)\right)\left(Y_{j, t_{2}}-E\left(Y_{j, t_{2}}\right)\right)\left|Y_{t_{1}-1}-Y_{t_{2}-1}\right|_{p} \\
\left(U_{2, n}\right)_{i j}=-\frac{1}{(n-1)(n-2)} \sum_{t_{1}=2}^{n} \sum_{t_{2} \neq t_{1}}\left(Y_{i, t_{1}}-E\left(Y_{i, t_{1}}\right)\right)\left(E\left(Y_{j, t_{2}}\right)-\left(\bar{Y}_{n-1}\right)_{j}\right)\left|Y_{t_{1}-1}-Y_{t_{2}-1}\right|_{p}
\end{gathered}
$$




$$
\begin{aligned}
& \left(U_{3, n}\right)_{i j}=-\frac{1}{(n-1)(n-2)} \sum_{t_{1}=2}^{n} \sum_{t_{2} \neq t_{1}}\left(E\left(Y_{i, t_{1}}\right)-\left(\bar{Y}_{n-1}\right)_{i}\right)\left(Y_{j, t_{2}}-E\left(Y_{j, t_{2}}\right)\right)\left|Y_{t_{1}-1}-Y_{t_{2}-1}\right|_{p} \\
& \left(U_{4, n}\right)_{i j}=-\frac{1}{(n-1)(n-2)} \sum_{t_{1}=2}^{n} \sum_{t_{2} \neq t_{1}}\left(E\left(Y_{i, t_{1}}\right)-\left(\bar{Y}_{n-1}\right)_{i}\right)\left(E\left(Y_{j, t_{2}}\right)-\left(\bar{Y}_{n-1}\right)_{j}\right)\left|Y_{t_{1}-1}-Y_{t_{2}-1}\right|_{p}
\end{aligned}
$$

and $\left(U_{1, n}\right)_{i, j}$ is a $U$-statistic of order 2 for the stationary time series $\left\{Z_{t}=\left(Y_{t}^{T}, Y_{t-1}^{T}\right)^{T}\right\}$. The kernel function for $\left(U_{1, n}\right)_{i j}$ is

$$
g\left(Z_{2}, Z_{2}^{\prime}\right)=-\left\{Y_{i, 2}-E\left(Y_{i, 2}\right)\right\}\left\{Y_{j, 2}^{\prime}-E\left(Y_{j, 2}^{\prime}\right)\right\}\left|Y_{1}-Y_{1}^{\prime}\right|_{p},
$$

where $Z_{2}=\left(Y_{2}^{T}, Y_{1}^{T}\right)^{T}$ and $Z_{2}^{\prime}=\left(Y_{2}^{\prime T}, Y_{1}^{\prime T}\right)^{T}$. Under our condition (C2), we have that $E\left(\left|g\left(Z_{2}, Z_{2}^{\prime}\right)\right|^{2+\delta}\right)<\infty$ and $E\left(\left|g\left(Z_{2}, Z_{2+k}\right)\right|^{2+\delta}\right), k=1, \cdots, n$ is bounded by using Cauchy-Swartz inequality. It then follows from Theorem 1 in Yoshihara (1976) that $\left|\left(U_{1, n}\right)_{i j}-M D D M\left(Y_{t} \mid Y_{t-1}\right)_{i j}\right|^{2}=O_{p}\left(n^{-1}\right)$ for $i, j=1, \cdots, p$. Since $\left|E\left(Y_{i, t_{1}}\right)-\left(\bar{Y}_{n-1}\right)_{i}\right|=$ $O_{p}\left(n^{-1 / 2}\right)$ for $i=1, \cdots, p,\left(U_{2, n}\right)_{i, j},\left(U_{3, n}\right)_{i, j},\left(U_{4, n}\right)_{i, j}$ are $O_{p}\left(n^{1 / 2}\right)$. Thus, these facts yield (2). Then the conclusion that $\widehat{\lambda}_{j}-\lambda_{j}=O_{p}\left(n^{-1 / 2}\right), j=1,2, \cdots, s$ follows since $\left|\operatorname{tr}\left(\gamma_{j} \gamma_{j}^{T}\left\{\widehat{\Gamma}_{1}-\Gamma_{1}\right\}\right)\right| \leq\left\|\gamma_{j}\right\|^{2}\left\|\widehat{\Gamma}_{1}-\Gamma_{1}\right\|_{2}=O_{p}\left(n^{-1 / 2}\right)$ and $\left|R_{1}\right|=O_{p}\left(n^{-1}\right)$ under condition (C1).

Regarding the eigenvector, we apply part (b) of that lemma and get that for $j=$ $1, \cdots, s$,

$$
\widehat{\gamma}_{j}-\gamma_{j}=-S_{j}\left(\Gamma_{1}\right)\left(\widehat{\Gamma}_{1}-\Gamma_{1}\right) \gamma_{j}+R_{3},
$$

where $S_{j}\left(\Gamma_{1}\right)=\sum_{h \neq j} \frac{1}{\lambda_{h}-\lambda_{j}} \gamma_{h} \gamma_{h}^{T}$ and $\left\|R_{3}\right\|_{2} \leq \frac{6\left\|\widehat{\Gamma}_{1}-\Gamma_{1}\right\|_{2}^{2}}{\min _{\lambda \in E G\left(\Gamma_{1}\right), \lambda \neq \lambda_{j}}\left|\lambda-\lambda_{j}\right|^{2}}$. Then $\sqrt{n}\left\|R_{3}\right\|_{2}=$ $O_{p}\left(n^{-1 / 2}\right)$ and $\left\|-S_{j}\left(\Gamma_{1}\right)\left(\widehat{\Gamma}_{1}-\Gamma_{1}\right) \gamma_{j}\right\|_{2}^{2}=\sum_{h \neq j} \frac{\left\{\gamma_{h}^{T}\left(\widehat{\Gamma}_{1}-\Gamma_{1}\right) \gamma_{j}\right\}^{2}}{\left(\lambda_{h}-\lambda_{j}\right)^{2}}=O_{p}\left(n^{-1}\right)$ which yields that $\left\|\widehat{\gamma}_{j}-\gamma_{j}\right\|_{2}=O_{p}\left(n^{-1 / 2}\right)$.

To show (ii), we note that part (a) of Lemma A1 of Kneip and Utikal (2001) implies that

$$
\sum_{j=s+1}^{p}\left(\widehat{\lambda}_{j}-\lambda_{j}\right)=\operatorname{tr}\left(\gamma_{s+1} \gamma_{s+1}^{T}\left\{\widehat{\Gamma}_{1}-\Gamma_{1}\right\}\right)+R_{2}
$$

where $\left|R_{2}\right| \leq \min (p-s, s) \frac{6\left\|\widehat{\Gamma}_{1}-\Gamma_{1}\right\|_{2}^{2}}{\min _{\lambda \in E G\left(\Gamma_{1}\right), \lambda \neq \lambda_{s+1}}\left|\lambda-\lambda_{s+1}\right|}=O_{p}\left(n^{-1}\right)$. Furthermore, we write 


$$
\begin{aligned}
& \operatorname{tr}\left(\gamma_{s+1} \gamma_{s+1}^{T}\left\{\widehat{\Gamma}_{1}-\Gamma_{1}\right\}\right)=\gamma_{s+1}^{T} \widehat{\Gamma}_{1} \gamma_{s+1}-\gamma_{s+1}^{T} \Gamma_{1} \gamma_{s+1}=\frac{n-2}{(n-1)}\left(V_{1, n}+V_{2, n}+V_{3, n}+V_{4, n}\right), \text { where } \\
& V_{1, n}=\frac{-1}{(n-1)(n-2)} \sum_{t_{1}=2}^{n} \sum_{t_{2} \neq t_{1}}\left\{\gamma_{s+1}^{T} Y_{t_{1}}-E\left(\gamma_{s+1}^{T} Y_{t_{1}}\right)\right\}\left\{\gamma_{s+1}^{T} Y_{t_{2}}-E\left(\gamma_{s+1}^{T} Y_{t_{2}}\right)\right\}\left|Y_{t_{1}-1}-Y_{t_{2}-1}\right|_{p} \\
& V_{2, n}=\frac{-1}{(n-1)(n-2)} \sum_{t_{1}=2}^{n} \sum_{t_{2} \neq t_{1}}\left\{\gamma_{s+1}^{T} Y_{t_{1}}-E\left(\gamma_{s+1}^{T} Y_{t_{1}}\right)\right\}\left\{E\left(\gamma_{s+1}^{T} Y_{t_{2}}\right)-\gamma_{s+1}^{T} \bar{Y}_{n-1}\right\}\left|Y_{t_{1}-1}-Y_{t_{2}-1}\right|_{p} \\
& V_{3, n}=\frac{-1}{(n-1)(n-2)} \sum_{t_{1}=2}^{n} \sum_{t_{2} \neq t_{1}}\left\{E\left(\gamma_{s+1}^{T} Y_{t_{1}}\right)-\gamma_{s+1}^{T} \bar{Y}_{n-1}\right\}\left\{\gamma_{s+1}^{T} Y_{t_{2}}-E\left(\gamma_{s+1}^{T} Y_{t_{2}}\right)\right\}\left|Y_{t_{1}-1}-Y_{t_{2}-1}\right|_{p} \\
& V_{4, n}=\frac{-1}{(n-1)(n-2)} \sum_{t_{1}=2}^{n} \sum_{t_{2} \neq t_{1}}\left\{E\left(\gamma_{s+1}^{T} Y_{t_{1}}\right)-\gamma_{s+1}^{T} \bar{Y}_{n-1}\right\}\left\{E\left(\gamma_{s+1}^{T} Y_{t_{2}}\right)-\gamma_{s+1}^{T} \bar{Y}_{n-1}\right\}\left|Y_{t_{1}-1}-Y_{t_{2}-1}\right|_{p}
\end{aligned}
$$

Since $\lambda_{s+1}=0, \operatorname{MDD}\left(\gamma_{s+1}^{T} Y_{2} \mid Y_{1}\right)=0$, i.e., $E\left(\gamma_{s+1}^{T} Y_{2} \mid Y_{1}\right)=E\left(\gamma_{s+1}^{T} Y_{2}\right)$ almost surely.

This implies that $V_{1, n}$ is a degenerate U-statistic of order 1. Thus

$$
\begin{aligned}
E\left(V_{1, n}^{2}\right)= & O\left(n^{-4}\right) \sum_{t_{1}=2}^{n} \sum_{t_{3}=2}^{n} \sum_{t_{2}=2}^{t_{1}-1} \sum_{t_{4}=2}^{t_{3}-1} E\left\{\left\{\gamma_{s+1}^{T} Y_{t_{1}}-E\left(\gamma_{s+1}^{T} Y_{t_{1}}\right)\right\}\left\{\gamma_{s+1}^{T} Y_{t_{2}}-E\left(\gamma_{s+1}^{T} Y_{t_{2}}\right)\right\}\right. \\
& \left.\left\{\gamma_{s+1}^{T} Y_{t_{3}}-E\left(\gamma_{s+1}^{T} Y_{t_{3}}\right)\right\}\left\{\gamma_{s+1}^{T} Y_{t_{4}}-E\left(\gamma_{s+1}^{T} Y_{t_{4}}\right)\right\}\left|Y_{t_{1}-1}-Y_{t_{2}-1}\right|_{p}\left|Y_{t_{3}-1}-Y_{t_{4}-1}\right|_{p}\right\} \\
= & O\left(n^{-4}\right) \sum_{t_{1}=2}^{n} \sum_{t_{2}=2}^{t_{1}-1} \sum_{t_{4}=2}^{t_{1}-1} E\left\{\left\{\gamma_{s+1}^{T} Y_{t_{1}}-E\left(\gamma_{s+1}^{T} Y_{t_{1}}\right)\right\}^{2}\left\{\gamma_{s+1}^{T} Y_{t_{2}}-E\left(\gamma_{s+1}^{T} Y_{t_{2}}\right)\right\}\right. \\
& \left.\left\{\gamma_{s+1}^{T} Y_{t_{4}}-E\left(\gamma_{s+1}^{T} Y_{t_{4}}\right)\right\}\left|Y_{t_{1}-1}-Y_{t_{2}-1}\right|_{p}\left|Y_{t_{1}-1}-Y_{t_{4}-1}\right|_{p}\right\} \\
= & O\left(n^{-4}\right) \sum_{t_{1}=2}^{n} \sum_{t_{2}=2}^{t_{1}-1} \sum_{t_{4}=2}^{t_{2}} E\left\{\left\{\gamma_{s+1}^{T} Y_{t_{1}}-E\left(\gamma_{s+1}^{T} Y_{t_{1}}\right)\right\}^{2}\left\{\gamma_{s+1}^{T} Y_{t_{2}}-E\left(\gamma_{s+1}^{T} Y_{t_{2}}\right)\right\}\right. \\
& \left.\left\{\gamma_{s+1}^{T} Y_{t_{4}}-E\left(\gamma_{s+1}^{T} Y_{t_{4}}\right)\right\}\left|Y_{t_{1}-1}-Y_{t_{2}-1}\right|_{p}\left|Y_{t_{1}-1}-Y_{t_{4}-1}\right|_{p}\right\}
\end{aligned}
$$

To simplify the notation, we denote $H\left(t_{1}, t_{1}-1, t_{2}, t_{2}-1, t_{4}, t_{4}-1\right)=E\left(\xi\left(t_{1}, t_{1}-1, t_{2}, t_{2}-\right.\right.$ 1) $\left.\xi\left(t_{1}, t_{1}-1, t_{4}, t_{4}-1\right)\right)$, where

$$
\begin{aligned}
& \xi\left(t_{1}, t_{1}-1, t_{2}, t_{2}-1\right)=\left\{\gamma_{s+1}^{T} Y_{t_{1}}-E\left(\gamma_{s+1}^{T} Y_{t_{1}}\right)\right\}\left\{\gamma_{s+1}^{T} Y_{t_{2}}-E\left(\gamma_{s+1}^{T} Y_{t_{2}}\right)\right\}\left|Y_{t_{1}-1}-Y_{t_{2}-1}\right|_{p} \\
& \xi\left(t_{1}, t_{1}-1, t_{4}, t_{4}-1\right)=\left\{\gamma_{s+1}^{T} Y_{t_{1}}-E\left(\gamma_{s+1}^{T} Y_{t_{1}}\right)\right\}\left\{\gamma_{s+1}^{T} Y_{t_{4}}-E\left(\gamma_{s+1}^{T} Y_{t_{4}}\right)\right\}\left|Y_{t_{1}-1}-Y_{t_{4}-1}\right|_{p}
\end{aligned}
$$

Then $E\left(V_{1, n}^{2}\right)=O\left(n^{-4}\right) \sum_{t_{1}=2}^{n} \sum_{t_{2}=2}^{t_{1}-1} \sum_{t_{4}=2}^{t_{2}} H\left(t_{1}, t_{1}-1, t_{2}, t_{2}-1, t_{4}, t_{4}-1\right)$. Write

$$
\begin{aligned}
& H\left(t_{1}, t_{1}-1, t_{2}, t_{2}-1, t_{4}, t_{4}-1\right)=E\left(\xi\left(t_{1}, t_{1}-1, t_{2}, t_{2}-1\right) \xi\left(t_{1}, t_{1}-1, t_{4}, t_{4}-1\right)\right) \\
= & E\left[E\left\{\xi\left(t_{1}, t_{1}-1, t_{2}, t_{2}-1\right) \xi\left(t_{1}, t_{1}-1, t_{4}, t_{4}-1\right) \mid \mathcal{F}_{t_{4}}, Y_{t_{1}}, Y_{t_{1}-1}\right\}\right] \\
= & E\left[\xi\left(t_{1}, t_{1}-1, t_{4}, t_{4}-1\right) E\left\{\xi\left(t_{1}, t_{1}-1, t_{2}, t_{2}-1\right) \mid \mathcal{F}_{t_{4}}, Y_{t_{1}}, Y_{t_{1}-1}\right\}\right]
\end{aligned}
$$


Under the $m$-dependence assumption for $\left\{Y_{t}\right\}$, we shall show that $H\left(t_{1}, t_{1}-1, t_{2}, t_{2}-\right.$ $\left.1, t_{4}, t_{4}-1\right)=0$ whenever $\left|\left(t_{2}-1\right)-t_{4}\right|>m$ and $\left|\left(t_{1}-1\right)-t_{2}\right|>m$. To see this, we note that

$$
\begin{aligned}
& E\left\{\xi\left(t_{1}, t_{1}-1, t_{2}, t_{2}-1\right) \mid \mathcal{F}_{t_{4}}, Y_{t_{1}}, Y_{t_{1}-1}\right\} \\
= & E\left\{\left\{\gamma_{s+1}^{T} Y_{t_{1}}-E\left(\gamma_{s+1}^{T} Y_{t_{1}}\right)\right\}\left\{\gamma_{s+1}^{T} Y_{t_{2}}-E\left(\gamma_{s+1}^{T} Y_{t_{2}}\right)\right\}\left|Y_{t_{1}-1}-Y_{t_{2}-1}\right|{ }_{p} \mid \mathcal{F}_{t_{4}}, Y_{t_{1}}, Y_{t_{1}-1}\right\} \\
= & \left\{\gamma_{s+1}^{T} Y_{t_{1}}-E\left(\gamma_{s+1}^{T} Y_{t_{1}}\right)\right\} E\left\{\left\{\gamma_{s+1}^{T} Y_{t_{2}}-E\left(\gamma_{s+1}^{T} Y_{t_{2}}\right)\right\}\left|Y_{t_{1}-1}-Y_{t_{2}-1}\right|_{p} \mid \mathcal{F}_{t_{4}}, Y_{t_{1}}, Y_{t_{1}-1}\right\} \\
= & \left\{\gamma_{s+1}^{T} Y_{t_{1}}-E\left(\gamma_{s+1}^{T} Y_{t_{1}}\right)\right\} \times \\
& E\left[E\left\{\left\{\gamma_{s+1}^{T} Y_{t_{2}}-E\left(\gamma_{s+1}^{T} Y_{t_{2}}\right)\right\}\left|Y_{t_{1}-1}-Y_{t_{2}-1}\right|_{p} \mid Y_{t_{2}-1}, \mathcal{F}_{t_{4}}, Y_{t_{1}}, Y_{t_{1}-1}\right\} \mid \mathcal{F}_{t_{4}}, Y_{t_{1}}, Y_{t_{1}-1}\right] \\
= & \left\{\gamma_{s+1}^{T} Y_{t_{1}}-E\left(\gamma_{s+1}^{T} Y_{t_{1}}\right)\right\} \times \\
& E\left[\left|Y_{t_{1}-1}-Y_{t_{2}-1}\right|_{p} E\left\{\left\{\gamma_{s+1}^{T} Y_{t_{2}}-E\left(\gamma_{s+1}^{T} Y_{t_{2}}\right)\right\} \mid Y_{t_{2}-1}, \mathcal{F}_{t_{4}}, Y_{t_{1}}, Y_{t_{1}-1}\right\} \mid \mathcal{F}_{t_{4}}, Y_{t_{1}}, Y_{t_{1}-1}\right]
\end{aligned}
$$

Since $\lambda_{s+1}=0$, which implies that $E\left(\gamma_{s+1}^{T} Y_{t_{2}} \mid Y_{t_{2}-1}\right)=E\left(\gamma_{s+1}^{T} Y_{t_{2}}\right)$ almost surely. Due to the independence between $Y_{t_{2}}$ and $\left(\mathcal{F}_{t_{4}}, Y_{t_{1}}, Y_{t_{1}-1}\right)$ under the $m$-dependence assumption, we can derive that

$E\left\{\left\{\gamma_{s+1}^{T} Y_{t_{2}}-E\left(\gamma_{s+1}^{T} Y_{t_{2}}\right)\right\} \mid Y_{t_{2}-1}, \mathcal{F}_{t_{4}}, Y_{t_{1}}, Y_{t_{1}-1}\right\}=E\left\{\left\{\gamma_{s+1}^{T} Y_{t_{2}}-E\left(\gamma_{s+1}^{T} Y_{t_{2}}\right)\right\} \mid Y_{t_{2}-1}\right\}=0$,

which implies that $E\left\{\xi\left(t_{1}, t_{1}-1, t_{2}, t_{2}-1\right) \mid \mathcal{F}_{t_{4}}, Y_{t_{1}}, Y_{t_{1}-1}\right\}=0$ and $H\left(t_{1}, t_{1}-1, t_{2}, t_{2}-\right.$ $\left.1, t_{4}, t_{4}-1\right)=0$.

Under the finite 6th moment assumption for $Y_{t}$, it follows from Cauchy-Swartz inequality that $\left|H\left(t_{1}, t_{1}-1, t_{2}, t_{2}-1, t_{4}, t_{4}-1\right)\right| \leq C$ for any $\left(t_{1}, t_{2}, t_{4}\right)$. Thus we have

$$
\begin{aligned}
E\left(V_{1, n}^{2}\right)= & O\left(n^{-4}\right) \sum_{t_{1}=2}^{n} \sum_{\left|\left(t_{1}-1\right)-t_{2}\right| \leq m} \sum_{t_{4}=2}^{t_{2}} H\left(t_{1}, t_{1}-1, t_{2}, t_{2}-1, t_{4}, t_{4}-1\right) \\
& +O\left(n^{-4}\right) \sum_{t_{1}=2}^{n} \sum_{\left|\left(t_{1}-1\right)-t_{2}\right|>m} \sum_{\left|\left(t_{2}-1\right)-t_{4}\right| \leq m} H\left(t_{1}, t_{1}-1, t_{2}, t_{2}-1, t_{4}, t_{4}-1\right)=O\left(n^{-2}\right),
\end{aligned}
$$

which yields $V_{1, n}=O_{p}\left(n^{-1}\right)$. By a similar but simpler argument, we can show that $V_{j, n}=O_{p}\left(n^{-1}\right)$ for $j=2,3,4$. Hence $\sum_{j=s+1}^{p}\left(\widehat{\lambda}_{j}-\lambda_{j}\right)=O_{p}\left(n^{-1}\right)$ which implies that $\widehat{\lambda}_{j}-\lambda_{j}=O_{p}\left(n^{-1}\right)$ for $j=s+1, \cdots, p$. The proof is now complete. 
Proof of Proposition 4.1: If $\left(\epsilon_{t}, \mathcal{F}_{t}\right)$ is a martingale difference sequence, it implies that $E\left(\epsilon_{t} \mid \mathcal{F}_{t-1}\right)=0$, which leads to

$$
E\left(Y_{t} \mid \mathcal{F}_{t-1}\right)=E\left(A X_{t}+\epsilon_{t} \mid \mathcal{F}_{t-1}\right)=A E\left(X_{t} \mid \mathcal{F}_{t-1}\right)
$$

The conclusion follows by letting $Z_{t}=E\left(X_{t} \mid \mathcal{F}_{t-1}\right)$ and $\mathcal{A}=A$.

\section{References}

Kneip, A. and Utikal, K. J. (2001) Inference for density families using functional principal component analysis. Journal of the American Statistical Association, 96, 519-542.

Székely, G. J., Rizzo, M. L. and Bakirov, N. K. (2007) Measuring and testing independence by correlation of distances. Annals of Statistics, 35, 2769-2794.

Yoshihara, K. (1976) Limiting behaviour of U-statistics for stationary absolutely regular processes. Z. Wahrscheinlichkeitstheorie verw. Gebiete., 35, 237-252. 